\title{
Association Analysis of Molecular Markers with Essential Agronomic Traits under Normal and Salt Stress in Wide Germplasm of Foxtail Millet (Setaria Italica L.)
}

\section{Mehdi Yazdizadeh}

University of Zabol

Ghasem Mohammadi-Nejad ( $D$ Mohammadinejad@uk.ac.ir)

Shahid Bahonar University of Kerman https://orcid.org/0000-0002-5767-9734

\section{Leila Fahmideh}

Gorgan University of Agricultural Sciences and Natural Resources

Fatemeh Ebrahimi

Shahid Bahonar University Faculty of Agriculture

Mahmood Solouki

University of Zabol

\section{Babak Nakhoda}

ABRII: Agricultural Biotechnology Research Institute of Iran

\section{Research Article}

Keywords: AFLP Marker, Agronomic Traits, Genetic variability, Linkage disequilibrium , Marker stability

Posted Date: August 11th, 2021

DOI: https://doi.org/10.21203/rs.3.rs-739296/v1

License: (c) (i) This work is licensed under a Creative Commons Attribution 4.0 International License.

Read Full License 


\section{Abstract}

Food security and nutrition concerns are putting an ancient, climate-smart grain back on our plates; Farm to fork, there has been a revival of interest in millet. Foxtail millet, as a multi-purpose product, has nutritious and medicinal potentials. This research is aimed at identifying combined markers as well as stable associations between such markers and the investigated agronomic traits in a wide range of foxtail millet germplasms under normal and salinity-stress conditions. In this context, association analysis has been conducted among 14 agronomic traits and 331 polymorphic AFLP markers generated by 12 primer combinations in 134 foxtail millet genotypes. Based on the analysis of population structure, the foxtail millet genotypes were divided into six subpopulations. The results showed that a number of markers had stable and significant associations under both normal and salinity-stress conditions with agronomic traits. The primer combinations had high polymorphic percentage, diversity indices were highly reliable and revealed significant genetic variability among the genotypes. Their PIC, MI and Shannon's indices were also highly reliable and revealed significant genetic variability among the genotypes. Since the markers introduced in this research have stable and strong associations with the investigated traits under normal and salinity stress conditions, they can be suitable candidates' in future marker-assisted breeding to improve salinity- resistance genotypes of foxtail millet in arid and semiarid areas.

\section{Introduction}

Environmentally induced stresses are considered influential parameters on the growth rate and productivity of plants. Climate change effects such as water scarcity, increasing salinity of agricultural lands, heightened intensity and duration of drought periods as well as the rising need to herbal products lead to soil desalination, alkalization and salinity of agricultural lands. This increasing salinity of agricultural lands would have devastating global effects. It is expected that $30 \%$ of such lands would become uncultivable in the next twenty-five years. (Kapoor et al., 2013) Furthermore, by the middle of 21st century, more than $50 \%$ of arable lands would no longer be cultivable. Therefore, increasing attention has been paid to the saline agriculture (Kapoor et al., 2013; Ahmed et al., 2016). Environmental stresses contribute to a wide range of reactions in plants such as variation in change genes expression, cell metabolism, plant growth and yield (Reddy et al., 2004).

Population growth and the decreasing quality of agricultural lands caused by salinity have prompted researchers to develop salinity-resistance plants via genetic improvement (Munns et al., 2006). This is while little investigation has been carried out on the millet as one those plants compatible with such conditions. This species belongs to the Paniceae family in the Panicoideae subfamily. As an advantage, it can be cultivated once it is too late for other plants to be farmed (Baker, 2003). Containing of small genome $(\sim 515 \mathrm{Mb} ; 2 \mathrm{n}=2 \mathrm{x}=18)$ makes foxtail millet as a model in monocot plant. Its physiological properties make it appropriate for hot climates; therefore, it requires treatment in hot weather. As an ancient grain crop, its historical reports date back to 7400 and even 7935 years ago (Li and Wu, 1996; 
Foxtail millet has a short growth period (60-90 days) and is considered suitable for harsh environmental conditions such as hot climate, uncultivable lands and water scarcity. Moreover, it has acceptable yield despite unsuitable conditions (Zhang et al., 2007). Foxtail millet, as a multi-purpose crop, enjoys nutritious and remedial properties and has higher nutrients compared to the ordinary grains (Saha et al., 2016). It also contains the highest level of protein $(305.8 \mathrm{mg} / \mathrm{g})$ compared to other crops such as wheat, rice and barely. Moreover, it has high fiber content (as $\beta$-glucans, $42.6 \%$ ) as well as precursors including minerals (3.3 mg/g), iron (0.3 mg/g) and calcium (0.3 mg/g) (Rao et al., 2011; Amadouv et al., 2011; Saha et al., 2016). Foxtail millet is used as grain and forage in poultry farming and livestock industries. Foxtail millet has considerable protein content (12.30 gr) compared to sorghum (9.97gr), barely $(10.72$ $\mathrm{gr})$, rice $(8.33 \mathrm{gr})$ and corn $(8.80 \mathrm{gr})$. Moreover, foxtail millet has higher vitamins than ordinary cereals. For example, it has higher amounts of B1 $(0.59 \mathrm{mg}), \mathrm{B} 2(0.11 \mathrm{mg}), \mathrm{B} 3(3.20 \mathrm{mg})$ and B5 $(0.82 \mathrm{mg})$ vitamins than major cereals such as barely with respective values of $0.33,0.10,1.73$ and $0.80 \mathrm{mg}$. The B1, B2, B3 and $B 5$ vitamins found in rice are $0.16,0.05,2.53$ and $0.42 \mathrm{mg}$, respectively. Besides, the corresponding values of vitamins B1, B2, B3 and B5 found in corn are $0.33,0.09,2.69,0.34 \mathrm{mg}$. Therefore, foxtail millet has superiority over such major cereals (Dayakar et al., 2017). Foxtail millet is considered a source of energy for pregnant women, nurses, kids and diabetic patients (Arora and Wu, 2002; Dwivedi et al., 2012). The germinated seeds of foxtail millet cultivars, the yellow seeds in particular, have high medical properties which are used to treat diseases such as dyspepsia, celiac, poor digestion, etc (Singh et al., 2016). In China, Japan, Korea and East Asia, it is utilized in confectionary industry to produce edible dishes as well as baking cakes and cookies and making purees. Moreover, it is used in alcohol and fermentation industries to produce malt and fermented beverages (Ang et al., 1999). Compared to major cereal crops like rice and wheat, the biological value of digestible protein found in foxtail millet is high as it contains seven out of the eight essential amino acids (Zhang et al., 2007). They also contain 2.5 times more edible fibers than rice. Moreover, their bran contains $9.4 \%$ crude oil enriched with linoleic $(66.5 \%)$ and oleic (13.0\%) acids (Liang et al 2010; Dwivedi et al., 2012), along with a considerable amount of fibers (42.56\%) (Rao et al., 2011). Besides, the huge contribution of these grains to the Chinese prehistoric civilization rendered them a special status among the "Five Grains" comprised of foxtail millet, proso millet, rice, soybean and wheat (Austin, 2006).

Improving salinity-resistance genotypes with the purpose of increasing crops yield in normal and lowyield soils, such as saline and alkaline ones, is among the major objectives of breeding programs (Munns and Tester, 2008). To this end, understanding the genetic complexity of salt endurance in crop plants is necessary to develop improved genotypes through conventional breeding methods. Practical statistics is considered as an approach to determine the associations among genetic markers and phenotypic data via Genome-wide association study (GWAS) technique. Therefore, such approaches will further facilitate the use of genetic resources to improve crops (Morton et al., 2019). Since phenotype is influenced by the environment, DNA-based methods such as DNA marker- assisted techniques have opened a new outlook to the molecular description of complex traits. Molecular marker is a certain DNA sequence which can easily be detected and its heredity is simply visible. These markers are used based on the natural DNA nolvmnrnhicm SSR RAPD $\triangle F I P$ and RFI P are among such markers. The AFLP marker has high Loading [MathJax]/jax/output/CommonHTML/jax.js 
reproducibility and sensitivity for finding polymorphism at various levels of the DNA sequence. Moreover, it does not need background knowledge on the initial sequence to design markers. Therefore, analysis of Amplified fragment length polymorphism (AFLP) is a strong and reliable technique compared to other DNA marker-assisted methods (Kumar et al., 2011).

The evaluation of association between polymorphism at DNA level and diverse phenotypic traits is considered an important tool in breeding programs (Sakiroglu et al., 2012). Studying the associations among molecular markers and agronomic traits has various applications such as (i) evaluation of polymorphism to detect informative markers for further breeding studies (ii) identification of favorable alleles in a germplasm set (iii) facilitating the determination of QTLs and (iv) endorsing the possible genes responsible for quantitative traits (Gebhardt et al., 2004).

The genomic loci affecting the quantitative traits are identified based on the linkage disequilibrium. This process is conducted using linkage and association analyses. The association analysis, extensively used to discover and identify the relationships between molecular markers and agronomic traits, is based on the linkage disequilibrium (LD) (Munns and Tester, 2008; Roy et al., 2011). The reliability of linkage analysis is influenced by factors such as the magnitude of polymorphism between two parents, the population size, the distribution of chiasma in a genome and the time needed to produce artificial populations. Nonetheless, association analysis does not require pure populations. Moreover, natural populations are used to find associations between markers and traits in this method. Since a recombination of these populations is created, the associations among the markers and the traits would be accurate and reliable (Parisseaux et al., 2004; Zhu et al., 2008). The population structure, effective on the level of LD, leads to a positive error, in other words, there might be no associations between the traits and markers but a fake one is created due to the structure of the population. Therefore, in order to avoid such fake affirmative associations among the markers and traits, the population structure of the germplasm must be explained before any association analyses. Software, called STRUCTURE, is used to determine and divide the population structure. This software, with high computational power, divides members into subpopulations. Then, it determines the membership probability of each individual. By correct assignment of these individuals to the subpopulations, association analysis will be conducted using an appropriate model (Aranzana et al., 2005).

Association analysis is performed using both general linear model (GLM) and mixed linear model (MLM) (Yu et al., 2006). In the GLM model, the subpopulations-related matrix (Q), the phenotypic and genotypic matrices are used. Moreover, the $Q$ matrix (structure matrix) is used as a covariance variable. This is while the kinship matrix $(\mathrm{K})$ is used along with membership matrix as well as the phenotypic and genotypic matrices in the MLM model (Pritchard et al., 2000; Yu et al., 2006; Kumar et al., 2015). In researches conducted on the genetic diversity of foxtail millet, those models developed based on the population structure and kinship relationship matrices could exhibit the real marker-trait relationships better than other ones, therefore, the fake affirmative associations decreased, significantly (Yu et al., 2006). 
Association analysis has successfully been employed to evaluate the relationships among molecular markers and agronomic traits in a wide range of crops. It has also used been to identify the genomic loci related to various traits (Berger et al., 2013). Gupta, S. et al (2014) studied the genetic diversity using 50 SSR markers, according to their investigation, 4 primers were associated with 184 foxtail genotypes via multiple factors.

In another investigation, 184 foxtail species were evaluated using SSR markers. The results indicated that 8 markers were placed on different chromosomes and had significant associations with 9 agronomic traits (Kumari et al., 2013). In a research conducted with the purpose of identifying genomic loci associated with the agronomic traits of pearl millet using SSR markers, it was found that plant height was associated with the LG7, LG4 and LG6 linkage groups. Moreover, the LG6 and LG7 alleles were associated with forage and seed yields, respectively. In another study, a number of QTLs including tillerring, plant height and panicle length were identified in agronomic traits of foxtail millet (Doust et al., 2004; Mauro-Herrera et al., 2013). Yazdizadeh et al (2020) used AFLP marker to perform association analysis on agronomic traits of 143 millet (Panicum miliaceum L.) cultivars. In this investigation, GLM and MLM models led to the identification of 866total band and 514 pollymorphic band with 11 combination markers associated with agronomic traits. Mehrabi et al (2020) used SNP marker to perform association analysis on agronomic traits of 112 durum wheat. In this investigation dentification $A$ total of 581 significant marker-trait associations were identified in all of the 14 chromosomes.

According to the literature, little investigation has been carried out on the association analysis of Setaria Italica ecotypes using a large number of markers under normal and salinity- stress conditions. Moreover, the AFLP markers and association analysis have a significant status in crops breeding programs. Therefore, this study has been conducted to identify the AFLP markers associated with important agronomic traits in a wide range of Iranian foxtail millet germplasms under both normal and salinitystress conditions. The targets of the present study are: (i) investigating the genetic diversity among genotypes based on evaluating those important traits proposed in previous researches under normal and salinity- stress conditions; (ii) evaluation of population genetic structures to detect essential marker-trait associations of the genotypes; (iii) the effectiveness of AFLP in recognizing the loci marker association with significant agronomic traits of Iranian species subjected to normal and salinity-stress conditions, separately; and (iv) determining the stability of respective loci markers with the desired agronomic traits corresponding to both conditions.

\section{Materials And Methods}

Plant materials

A total of 134 seeds of foxtail millet genotypes corresponding to a wide range of foxtail millet germplasms were procured by Iranian center of excellence for drought and salinity tolerant crops in Research and Technology Institute of Plant Production (RTIPP) belonged to Shahid Bahonar University of Korman_Iran /Cunnlomontary Tahlo 1) Tho research was conducted in two locations at RTIPP (ShahidLoading [MathJax]/jax/output/CommonHTML/jax.js 
Bahonar University of Kerman and Ekhtiyarabad fields). The experimental design was performed in a randomized complete block design with 3 replications under two conditions (normal and salinity) over a two year- long period (2017-2018).

\section{Test Location}

The fields of kerman used for the experiments are located at longitude $56^{\circ} 54^{\prime} \mathrm{E}$ and latitude $30^{\circ} 20^{\prime} \mathrm{N}$. They are situated at 1755 meters above the sea level. Also the field of Ekhtiyarabad used for the experiments are located at longitude $56^{\circ} 48^{\prime} \mathrm{E}$ and latitude $30^{\circ} 36^{\prime} \mathrm{N}$. They are situated at 1705 meters above the sea level. The soil has clayey loam tissue.

The field preparation operations such as plowing, weeding, fragmentation, fertilization and irrigation were regularly conducted at appropriate time intervals over the whole period of experiment. The soil $\mathrm{pH}$ was determined 7.41 and 8.2 in Kerman and Ekhtiyarabad fields, respectively. Moreover, the electrical conductivity of waters used in Kerman and Ekhtiyarabad fields was measured 2.5 and $8.7 \mathrm{dS} \mathrm{m}^{-1}$, respectively. The salt stress treatment began following irrigation with salty water in the germination stage and continued up to the end of harvesting process.

Due to the importance of seed germination under salinity, a separate experiment is done at Laboratory. Two separate tests were conducted under normal and salinity- stress conditions. Since the salinity of water used for irrigation purposes was evaluated 8.7, the seed germination percentage corresponding to salinity- stress conditions was determined in petri dish at an electrical conductivity of 8.7 while the seed germination percentage corresponding to normal conditions was determined in petri dish at an electrical conductivity of 2.5 .

Phenotypic evaluation

The traits evaluated in this investigation included seed germination (\%), plant height (cm), the number of leaves per plant, flag leaf length $(\mathrm{cm})$, flag leaf width $(\mathrm{cm})$, the number of tillers, panicle length $(\mathrm{cm})$, main panicle seed weight ( $\mathrm{g} \mathrm{m} 2-1)$, the number of plants on the line, 1000-seed weight, harvest index, forage yield ( $t$ ha- 1 ), biological yield ( $t$ ha- 1 ) and seed yield ( $t$ ha- 1 ). The last three traits including forage yield, biological yield and seed yield were initially measured based on ( $\mathrm{g} \mathrm{m} 2-1)$ and then converted to ton per hectare (t ha-1).

The analysis of variance (ANOVA) was conducted based on randomized complete block design (RCDB). The data were standardized for each trait and ANOVA was performed using SAS software ver 9.1 (SAS Institute 2004). The general heritability of the basic agronomic traits, under normal and salinity- stress conditions, was evaluated based on the Nyquist and Baker (1991) method:

$$
H^{2}=\sigma_{g}^{2} /\left(\sigma_{g}^{2}+\sigma_{g e}^{2} / e+\sigma_{\epsilon}^{2} / r e\right)
$$

1

Loading [MathJax]/jax/output/CommonHTML/jax.js

Page 6/24 
Where $\sigma_{\text {ge }}^{2}, \sigma^{2}$ and $\sigma_{\epsilon}^{2}$ are the genetic, genetic $\times$ environment interaction and environment variances, respectively. Moreover, e and $r$ indicate the number of environments and replications for each environment, respectively.

Molecular evaluation

In order to analyze molecular data, 20 AFLP primer combination markers were used. Then, 12 combinations with the highest polymorphic percentage were detected and evaluated (Table 1).

Table 1

Primer combinations used in the analysis of amplified fragment length polymorphism (AFLP) of foxtail millet.

\begin{tabular}{|lll|}
\hline Code of primer combination & Code Sequence for Msel & Code Sequence for EcoRI \\
\hline M14/E11 & Code Sequence for Msel & EcoRI Selective primer + AGC \\
\hline M59/E36 & Msel Selective Primer + CTG & EcoRI Selective primer + AGC \\
\hline M4/E10 & Msel Selective Primer + CTA & EcoRI Selective primer + AGC \\
\hline M3/E11 & Msel Selective Primer + CTT & EcoRI Selective primer + AGC \\
\hline M4/E11 & Msel Selective Primer + CAA & EcoRI Selective primer + AGC \\
\hline M59/E11 & Msel Selective Primer + CTT & EcoRI Selective primer + AGC \\
\hline M59/E10 & Msel Selective Primer + CTA & EcoRI Selective primer + AGC \\
\hline M4/E36 & Msel Selective Primer + CTA & EcoRI Selective primer + AGC \\
\hline M3/E36 & Msel Selective Primer + CTT & EcoRI Selective primer + AGC \\
\hline M14/E10 & Msel Selective Primer + CAA & EcoRI Selective primer + AGC \\
\hline M14/E36 & Msel Selective Primer + CTT & EcoRI Selective primer + AGC \\
\hline M3/E10 & Msel Selective Primer + CTT & EcoRI Selective primer + AGC \\
\hline
\end{tabular}

To extract DNA, samples were taken from the pristine leaves of five plants for each genotype. Having recorded the name as well as date of sampling on the packages containing those samples, they were put in liquid nitrogen and kept at $-80^{\circ} \mathrm{C}$ temperature. The DNAs were extracted from these leaves based on the Doyle and Doyle (1991) method. To evaluate the extracted DNAs, both quantitatively and qualitatively, $1 \%$ agarose gel and a spectrophotometer were used. The corresponding AFLP tests were conducted according to the Vos et al. (1995) protocol. To this end, two types of restriction enzymes, namely Msel and EcoRI, were used. In the selective reproduction stage, 20 AFLP primer combinations markers were applied. Then, 12 combinations with the highest polymorphic percentage were selected for genotyping. In order to distinguish as well as high- precision observation of the bands, the QIAXEL DNA machine was used. This method is considered a replacement for the poly-acryl-amid gel technique. 
In this method, $15 \mu$ of the PCR product is required. The QIAXEL machine can separate twelve DNA specimens' parts with high resolution in three minutes. Moreover, this process can be performed on 96 specimens, simultaneously. The electrophoresis of the specimens was conducted using kit's High Resolution (QIAGEN, Hilden, Germany). Then, the bands were calculated using Biocalculator software. In order to evaluate the population structure, the achieved matrix was evaluated using Structure software v.2.3.4 for 10000 Bure-in and $100000 \mathrm{MCMC}$, both in admixture form. This process was performed for $\mathrm{K}$ $=2$ to $\mathrm{K}=10$ (with 5 iterations for each $\mathrm{K}$ value). Using the optimum $\mathrm{K}$ determined based on the Evano et al. (2005) method, the Q matrix was derived from the results of population structure obtained by Structure software. To determine associations among the AFLP marker and morphologic traits, the Tassel software v. 2.1 was used. The association analysis was conducted using both GLM and MLM models. In the MLM model, the Q (population structure coefficients) and K (kinship matrix) matrices were combined to have more capability compared to linear modeling. It was found that this method is much better than the linear modeling's (GLM), commonly used in association analyses (Yu et al., 2006).

The optimum $\mathrm{K}$ was determined based on $\Delta \mathrm{K}$ achieved by the following equation:

$$
\Delta K=m|L "(K)| / \mathrm{s}[\mathrm{L}(\mathrm{K})]
$$

2

In the webpage of STRUCTURE HARVESTER processing, an optimum estimate of $\mathrm{K}$ is used to demonstrate the highest value of $\Delta \mathrm{K}$.

To determine marker-based indices, GenAlXe V6/5 programs were employed. Marker index (MI), Shannon's index $(\mathrm{H})$ and polymorphic information content (PIC) were among indices determined based on the following equations:

$$
P I C=1-p^{2}-q^{2}
$$

3

$$
M I=P I C \times \text { EquationNumberofpolymorphicloci }
$$

4

$$
H=-1 \times(p \times \ln (p)+q \times \ln (q))
$$

5

The symbols $p$ and $q$ denote prevailing and unvalued alleles, respectively.

Analysis of molecular variance (AMOVA), as a method to determine F-statistics among and within populations, was conducted using GenAlEx v6.5. The analogue stabilization index (PhiPT) statistics (analogy of FST, fixation index) was employed to estimate the genetic difference among subpopulations:

PhiPT = AP/ (WP + AP) (6) 
The symbols AP and WP indicate the approximate differences among and within populations, respectively.

\section{Results}

Phenotypic data analysis

Analysis of variance (ANOVA) was conducted using the PROC ANOVA procedure of SAS software v. 9.1. The results indicated that all genotypes, influenced by the genotypexenvironment interaction, They had significant difference in terms of all agronomic traits except for flag leaf length, flag leaf width, the number of tillers and panicle length under both normal and salinity-stress conditions. Moreover, all traits except for the flag leaf width were significant in terms of genotypes (Supplementary Table 2). This significant genetic diversity among some of the investigated genotypes provides an opportunity for selecting those morphologic traits leading to the increased efficiency of breeding programs. Under normal conditions, foxtail millet cultivars demonstrated a wide range of phenotypic variations for the investigated traits. For example, the seed germination varied from 60 to 80 percent. The plant height also ranged from 70 to $101 \mathrm{~cm}$. The highest level of germination (80\%) corresponded to the $\mathrm{G} 102$ genotype while the lowest value (60\%) was considered in G24 genotype. Moreover, the G24 and G102 genotypes had the highest $(101 \mathrm{~cm})$ and lowest $(70 \mathrm{~cm})$ plant height, respectively (data not shown). Additional file 2: Table S2 shows the general heritability to evaluate the traits of foxtail millet genotypes. The highest general heritability was observed for panicle length under normal (0.95) and salinity-stress conditions (0.87). This is while the lowest general heritability corresponded to the number of plants on a line under normal (0.19) and salinity-stress conditions (0.17) (Supplementary Table 2).

Allele diversity

Of the total 20 primer combinations used in this investigation, 12 primer combinations with the highest polymorphic bands were selected. Since small alleles are usually used in LD assessment of pairs of loci (Mohlke et al., 2001) those alleles with frequency of lower than 0.05 have been eliminated before any analyses. The 12 AFLP primer combinations used for 134 foxtail millet genotypes led to the identification of 331 polymorphic bands, i.e., an average of 27.58 polymorphic bands for each combination. The highest proportion of polymorphic bands to the total number of identified ones corresponded to the M59/E10 primer combination with 40 bands while the lowest one was observed in M3/E36 primer combination with 17 bands.

The average polymorphic information content (PIC) was determined 0.41. Furthermore, the highest level of PIC was related to M4/E10 with 0.68 and the lowest one corresponded to M59/E36 and M3/E36 primer combinations with 0.14 . The percentage range of polymorphism was between 41.46 (M3/E36 primer combination) and 80 (M59/E10) with an average value of 52.96. Regarding the high polymorphic percentage of M59/E10, M4/E10, M14/E11, M14/E10 and M4/E36 primer combinations, it is expected that they play an effective role in identifying and distinguishing foxtail millet genotypes. 
The marker index (MI) determines the effectiveness and capability of a marker based on the number of polymorphic genetic loci caused by that specific marker. The M4/E10 primer combination had the highest level of MI (23.80) while the M59/E36 and M3/E36 had the lowest value (2.21). Moreover, the M4/E10 and M3/E36 had the highest (1.17) and lowest (0.28) Shannon's index, respectively (Table 2).

Table 2

Statistical variance of 12 AFLP primer combinations for the 134 foxtail millets (Setaria italica L.) genotypes.

\begin{tabular}{|c|c|c|c|c|c|c|}
\hline $\begin{array}{l}\text { Primer } \\
\text { combination }\end{array}$ & $\begin{array}{l}\text { Total } \\
\text { Bands }\end{array}$ & $\begin{array}{l}\text { Polymorphic } \\
\text { bands }\end{array}$ & $\begin{array}{l}\text { Polymorphic } \\
\text { percentage }\end{array}$ & PIC & $\begin{array}{l}\text { Marker } \\
\text { Index }\end{array}$ & $\begin{array}{l}\text { Shannon } \\
\text { index }\end{array}$ \\
\hline M3/E10 & 50 & 26 & 52 & 0.35 & 9.10 & 0.66 \\
\hline M4/E10 & 50 & 35 & 70 & 0.68 & 23.80 & 1.17 \\
\hline M14/E10 & 50 & 32 & 64 & 0.61 & 19.52 & 1.04 \\
\hline M59/E10 & 50 & 40 & 80 & 0.59 & 23.60 & 1.10 \\
\hline M3/E11 & 50 & 24 & 48 & 0.35 & 8.40 & 0.65 \\
\hline M4/E11 & 50 & 27 & 54 & 0.36 & 9.72 & 0.68 \\
\hline M14/E11 & 50 & 35 & 70 & 0.47 & 16.45 & 0.86 \\
\hline M59/E11 & 50 & 26 & 52 & 0.49 & 12.74 & 0.85 \\
\hline M3/E36 & 41 & 17 & 41.46 & 0.13 & 2.21 & 0.28 \\
\hline M4/E36 & 50 & 31 & 62 & 0.4 & 12.40 & 0.75 \\
\hline M14/E36 & 50 & 21 & 42 & 0.39 & 8.19 & 0.69 \\
\hline M59/E36 & 32 & 17 & 53.13 & 0.13 & 2.21 & 0.29 \\
\hline Total & 573 & 331 & 635.46 & 4.84 & 146.13 & 8.79 \\
\hline Mean & 47.75 & 27.58 & 52.96 & 0.41 & 12.18 & 0.73 \\
\hline
\end{tabular}

Population Structure

The population structure was determined based on the molecular markers using non-parametric method introduced by Evano et al (2005). According to the results obtained by HARVESTER STRUCTURE, the highest level of $\Delta K$ corresponded to $K=6$. Figures $1-4$ show the four stages used to determine the real value of $\mathrm{K}$. 
Table 3 demonstrate the results of analysis of molecular variance (AMOVA) corresponding to six subpopulations derived from the population structure analysis. The results indicated that the variance among and within subpopulations contributed $3 \%$ and $97 \%$ of the total variance. Moreover, all PhiPT (analogue stabilization index) values were significant (0.03) (Table 3). On the other hand, the PhiPT values of each pair of sub-populations demonstrated significant differences among all subpopulations. Eventually, the required population structure $(\mathrm{Q})$ used for association analysis was established.

Table 3

Molecular variance (AMOVA) of association analysis of AFLP markers in six subpopulations derived from the 134 investigated foxtail millet (setaria italica L.) accessions using the structure software.

\begin{tabular}{|llllll|}
\hline Source of variation & Df & MS & Est. Var & $\%$ & PhiPT \\
\hline Among populations & 5 & 64.53 & 1.35 & $3 \%$ & $0.03^{\star *}$ \\
\hline Within populations & 128 & 40.61 & 40.61 & $97 \%$ & \\
\hline Total & 133 & - & - & $100 \%$ & \\
\hline$* *$ Significant at 0.01 levels. & & & & \\
\hline
\end{tabular}

Association analysis

In MLM method, the correlations among the markers and traits were evaluated using the data obtained by subpopulations-based membership matrix (Q), phenotypic data as well as the markers-related data derived from a multiple regression model. Moreover, the distribution of markers represented by determination coefficient (R2) was evaluated. According to the association analysis performed by the MLM model, the number of markers showing the significant relationship with the average of the investigated traits was determined 93 and 99 under normal and salinity stress conditions, respectively.

The association analysis of average traits using the GLM model ( $P+Q$ matrices) indicated that 114 and 107 markers were significant $(P<0.01)$ under normal and salinity-stress conditions, respectively (data not shown). According to the GLM model, the determination coefficient $\left(R^{2}\right)$ ranged from 9.05 to $28.23 \%$ under normal and 8.53 to $28.24 \%$ under salinity-stress conditions. Furthermore, the $\mathrm{R}^{2}$ value of model varied from 11.74 to $41.07 \%$ under normal and 15.06 to $41.06 \%$ under salinity-stress conditions.

According to the MLM model, the determination coefficient $\left(R^{2}\right)$ ranged from 5.4 to $15.38 \%$ under normal and 5.4 to $15.54 \%$ under salinity-stress conditions. Moreover, the $\mathrm{R}^{2}$ value of model varied from 40.12 to $62.89 \%$ under normal and 40.63 to $63.17 \%$ under salinity-stress conditions.

The results obtained by the MLM model indicated that the M4/E10-19, M14/E36-17, M14/E10-15, 
respective variations of $14.24 \%, 13.46 \%, 6.87 \%, 6.51 \%, 6.53 \%, 6.46 \%, 6.31 \%, 5.92 \%$ and $5.52 \%$ had the highest associations with seed germination under normal conditions. Moreover, the M4/E10-19, M14/E36-17, M59/E10-17, M14/E10-15, M14/E11-14, M59/E10-15, M3/E10-38, M14/E36-27 and M59/E36-4 markers with the corresponding variations of $13.04 \%, 12.47 \%, 7.30 \%, 6.82 \%, 6.35 \%, 6.21 \%$, $5.92 \%, 5.68 \%$ and $5.41 \%$ had strong associations with the same trait under salinity-stress conditions (Supplementary Table 3).

Based on the association analysis of foxtail millet, the M4/E10-9, M14/E36-14, M14/E11-14, M59/E1017, M59/36 - 4, M59/E10-15, M10/E14-15 and M3/E10-38 markers with a total variation of $66.63 \%$ demonstrated a strong association with the plant height under normal conditions. Furthermore, the M4/E10-19, M14/E36-17, M59/E10-17, M14/E11-14, M14/E10-15, M59/E36-4, M59/E10-15, M3/E10-38 and M14/E36-27 markers with the corresponding variations of $14.1 \%, 13.32 \%, 7.24 \%, 7.02 \%, 6.63 \%$, $6.39 \%, 6.30 \%, 6.03 \%$ and $5.84 \%$ displayed strong associations with plant height under salinity-stress conditions (Supplementary Table 3).

According to the MLM model, ten markers including M4/E10-19, M14/E36-17, M59/E10-17, M14/E11-14, M59/E10-15, M3/E10-38, M59/E36-4, M14/E10-15, M14/E36-27 and M59/E11-3 with a total variation of $76.70 \%$ had strong associations with the number of leaves per plant under normal conditions. Moreover, the M4/E10- 19, M14/E36-17, M59/E10-17, M14/E11-14, M59/E10-15, M3/E10-38, M59/E36-4, M14/E1015, M14/E36-27 and M59/E11- 3 markers with a total variation of $76.75 \%$ demonstrated meaningful associations with the same trait under salinity-stress conditions (Supplementary Table 3).

The results also revealed that seven markers including M14/E36-17, M14/E10-19, M59/E10-17, M59/E364, M14/E10-15, M14/E11-14 and M59/E10-10 with the corresponding variations of $13.71 \%, 11.83 \%$, $7.91 \%, 7.91 \%, 6.51 \%, 6.41 \%$ and $5.64 \%$ had strong relationships $(P<0.004)$ with the flag leaf length under normal conditions. This is while the M14/E36-17, M4/E10-19, M59/E10-17, M59/E36-4, M14/E11-14, M59/E10-10, M14/E36-27 and M14/E10-15 markers with the respective variations of $13.03 \%, 12.37 \%$, $9.03 \%, 7.74 \%, 6.43 \%, 5.96 \%, 5.94 \%$ and $5.82 \%$ had such relationships with the flag leaf length under salinity-stress conditions (Supplementary Table 3).

According to the MLM model, the M3/E11-13 and M59/E36-3 markers with a total variation of $15.88 \%$ demonstrated a strong association with the flag leaf width under normal conditions, while the M3/E11-13 and M4/E10-5 markers with the respective variations of 10.57 and $6.43 \%$ had such relationships with the flag leaf width under salinity-stress conditions (Supplementary Table 3 ).

This model further showed that the M14/E36-9, M4/E36-23 and M14/E11-15 markers with a total variation of $20.09 \%$ had strong relationships with the number of tillers under normal conditions. Besides, three markers including M14/E36-9, M4/E36-23 and M4/E10-23 with a total variation of $21.02 \%$ had significant associations $(P<0.006)$ with the number of tillers under salinity-stress conditions (Supplementary Table 3). 
The results also indicated that the M4/E10-19, M14/E36-17, M14/E11-14, M3/E10-38, M59/E36-4, M59/E10-15, M59/E10-17, M14/E10-44 markers with the respective variations of 15.38\%, 13.93\%, 7.94\%, $7.52 \%, 7.14 \%, 6.80 \%, 6.71 \%$ and $5.76 \%$ had considerable relationships with the panicle length under normal conditions. This is while the M4/E10-19, M14/E36-17, M59/E10-15, M59/E10-17, M59/E36-4, M14/E11-14 and M14/E10-15 markers with the respective variations of $15.54 \%, 13.34 \%, 7.30 \%, 7.19 \%$, $6.77 \%, 6.66 \%$, and $5.84 \%$ had such relationships with the panicle length under salinity-stress conditions (Supplementary Table 3).

The association analysis conducted on the foxtail millet genotypes using MLM model further showed that the M14/E36-14, M4/E10-19, M59/E36-27 and M59/E36-4 markers with a total variation of 33.77\% variation had significant associations with the seed yield under normal conditions. Moreover, M14/E3617, M4/E10-19, M59/E36-25, M59/E36-4, M59/E10-29 and M14/E11-25 markers with a total variation of $44.01 \%$ had considerable relationships with this particular trait under salinity-stress conditions (Supplementary Table 3).

The results also demonstrated that the M4/E10-19, M14/E36-14, M14/E11-14, M59/E10-17, M14/E10-15, M59/E10-15 and M59/E36-4 markers with an overall variation of $61.32 \%$ had considerable associations (Pcript $>$ ) with the number of plants on the line under normal conditions, while the M4/E10-19, M14/E36-17, M14/E11-14, M59/E10-17, M14/E10-15, M59/E36-4 and M59/E10-15 markers with a total variation of $61.49 \%$ had such relationships with this specific trait under salinity-stress conditions (Supplementary Table 3).

This model also indicated that the M14/E36-17, M3/E10-38, M4/E10-19, M14/E11-14, M59/E10-10, M59/E36-4, M59/E11-3 and M14/E36-27 markers with an overall variation of 76.91\% had significant associations with the forage yield under normal conditions. Moreover, the M14/E36-17, M4/E10-19, M14/E11-14, M3/E10-38, M59/E10-10, M3/E10-3 and M59/36 - 4 markers with a total variation of $62.07 \%$ had considerable relationships with the forage yield under salinity-stress conditions (Supplementary Table 3).

According to the MLM model, three markers including M59/E10-3, M59/E36-25 and M4/E11-3 with the corresponding values of $9.14 \%, 8.10 \%$ and $8.04 \%$ demonstrated significant associations $(P<0.005)$ with main panicle seed weight under normal conditions. This is while the M59/E10-3 and M59/E36-3 markers with a total variation of $17.04 \%$ demonstrated such relationships with this particular trait under salinitystress conditions (Supplementary Table 3).

In this model, the M14/E36-17, M4/E10-19, M59/E10-17, M14/E10-15, M59/E36-4, M14/E11-14 and M3/E10-38 markers with an overall variation of $53.45 \%$ displayed considerable associations with the 1000-seed weight under normal conditions. Moreover, the M14/E36-17, M4/E10-19, M3/E10-38, M59/E10-17, M14/E11-14, M59/E10-10, M59/E36-4 and M59/E11-3 markers with a total variation of $61.02 \%$ showed significant relationships $(P<0.0074)$ with this specific trait under salinity-stress conditions (Supplementary Table 3). 
The MLM model also showed that the M4/E10-19, M14/E36-17, M14/E11-14, M59/E10-17, M14/E10-15, M59/E10-15 and M59/E36-4 markers with the respective values of $13.95 \%, 12.92 \%, 7.64 \%, 7.49 \%, 6.98 \%$, $6.12 \%$ and $5.92 \%$ had strong associations with the harvest index under normal conditions. Furthermore, the M4/E10-19, M14/E36-17, M14/E11-14, M59/E10-17, M14/E10-15, M59/E36-4 and M59/E10-15 markers with a total variation of $61.19 \%$ had considerable relationships $(P<0.006)$ with this particular trait under salinity-stress conditions (Supplementary Table 3).

This model also indicated that the M4/E10-19, M14/E36-17, M59/E10-17, M14/E11-14, M14/E10-15, M59/E36-4 and M59/E10-15 markers with a total variation of $60.34 \%$ had considerable associations with the biological yield under normal conditions. This is while the M4/E10-19, M14/E36-17, M59/E10-17, M14/E11-14, M14/E10-15, M59/E36-4 and M59/E10-15 markers with a total variation of 60.57\% showed such associations with this specific trait under salinity-stress conditions (Supplementary Table 3).

To determine stable relationships, the association analysis of the foxtail millet genotypes using the MLM model was conducted for each location, separately. The results indicated that the M4/E10-19and M14/E36-17markers had significant and stable associations with the seed germination under normal conditions. Similar relationships were also observed between the M4/E10-9 marker and plant height, the M4/E10-19 and M14/E36-17markers and the number of leaves as well as the M14/E36-9 marker and the number of tillers under such conditions. Moreover, the M4/E10-19 and M14/E36-17 markers demonstrated considerable and stable associations with the seed and forage yields, 1000-seed weight, biological yield and harvest index under similar conditions. This is while the M4/E10-19 marker had significant and stable relationships with the seed germination percentage as well as the seed yield under salinity-stress conditions. Similar associations were also observed between the M4/E10-19 and M59/E10-9 markers and 1000-seed weight, the M59/E10-10 marker and forage yield, the M59/E10-9 marker and plant height as well as the M4/E10-19 marker and the number of leaves under similar conditions. Therefore, a number of markers demonstrated stable and significant associations with traits under both normal and salinity-stress conditions.

According to the results, a number of the investigated traits had associations with several traits. The highest level of associations was found in loci 17, 19 and 17 corresponding to the M14/E36, M4/E10 and M59 / E10 markers. The Significant common markers among a number of traits might be attributed to the pleiotropic effects or bonds of genomic loci affecting them (Jun et al., 2008). These pleiotropic effects have also been reported by other researchers (Zhang et al., 2015; Mwadzingeni et al., 2017).

In this research, the AFLP markers associated with important agronomic traits of foxtail millet were identified. These stable markers were determined from a total number of 134 foxtail millet genotypes under both normal and salinity-stress conditions.

\section{Discussion}

The germplasm evaluation of a specific plant species using molecular markers is considered a valuable Loading [MathJax]/jax/output/CommonHTML/jax.js lasm and identify suitable genotypes even under laboratory 
conditions. This is the first investigation on the application of association analysis to identify the molecular markers associated with important agronomic traits of a wide range of Iranian foxtail millet germplasm under normal and salinity-stress conditions.

Association analysis is a suitable technique to identify the genes' loci caused by salinity-stress as well as labeling different traits. It is also effective in marker-assisted methods used in foxtail millet under salinitystress conditions. Therefore, increasing knowledge in this field could be instrumental in protecting the effective germplasm and choosing the best one for breeding programs and other genetic researches (Mwadzingeni et al., 2017). Based on the analysis of phenotypic data, genotypes are significantly different in terms of the investigated traits. This difference indicates the presence of a considerable diversity in germplasm which in turn can facilitate selecting salinity- resistance genotypes.

The phenotypic analysis further shows that many traits are significantly influenced by the environment as well as the genotypes xenvironment interaction under both normal and salinity-stress conditions.

Therefore, investigating association analysis in different environments is highly significant. Moreover, the molecular analyses indicate high percentage of polymorphism, demonstrating the AFLP combinations employed in this investigation might be a powerful tool to distinguish salinity- resistance foxtail millet genotypes. These findings are in agreement with the results of other investigations conducted on foxtail millet (d'Ennequin et al., 2000; Gupta et al., 2011; Mohammadi-Nejad et al., 2017; Yazdizadeh et al., 2020).

In this research, the M59/E10, M4/E10, M14/E11, M14/E10 and M4/E36 primer combination has the highest polymorphic percentage as well as high PIC, MI and Shannon's indices. Therefore, this marker is considered as the most powerful combination to distinguish foxtail millet genotypes. Based on the analysis of population structure, the genotypes have been divided into six subpopulations with different genetic structures. The AMOVA results indicate that the genetic variance among subpopulations is very significant. These findings also demonstrated that the AFLP marker can be used to analyze populations which are in agreement with the results of Kumar et al., (2015). According to their investigation, the AFLP marker could be used as an index for genetic classification, construction a linkage maps, mapping traits and association study.

According to the association analyses under normal and salinity-stress conditions, the number of significant markers in the MLM has decreased compared to the GLM model. A combination of kinship and population structure in the MLM model would be effective to reduce fake affirmative associations, demonstrating some alleles identified by the GLM model could be attributed to the associations between genotypes and traits. These results are in agreement with the finding of Yu et al., (2006) and Dadras et al., (2014). Moreover, the determination coefficient obtained by the MLM has shown considerable reduction compared to the corresponding value in the GLM model. Therefore, using of AFLP markers along with MLM model is considered as a suitable method for future studies. These results are also consistent with the findings of Achleitner et al., (2008). They reported that combining Q and K in the MLM model could reduce determination coefficient and probably provide the best modification in population structures. Nonethelecs markers muct he verified hv examining their effectiveness on definitive goal-oriented Loading [MathJax]/jax/output/CommonHTML/jax.js

Page 15/24 
phenotypes among absolute populations with diverse genetic backgrounds (Collard et al., 2005). Those markers, showing the highest effect on the traits, would be the best possible candidates for future researches including the marker-assisted studies.

According to the association analysis using MLM model, the M4/E10-19 and M14/E36-17markers have stable and significant association with seed and forage yields, 1000-seed weight, biological yield and harvest index under normal conditions. Similar relationship has also been found between the M4/E10-19 marker and seed yield under salinity-stress conditions. Furthermore, the M4/E10-19 and M59/E10-9 markers have shown stable and significant association with the 1000-seed weight under similar conditions. The M59/E10-9 marker has also demonstrated a stable and considerable relationship with the seed and forage yields under salinity-stress conditions. Those cultivars with higher seed and forage yields would be more suitable for breeding programs of foxtail millet. Therefore, if these loci demonstrate to be effective on genetic control of such traits, they could be useful tools in molecular breeding programs of foxtail millet under salinity stress conditions. In this context, Shi et al., (2009) proposed using local QTLs to evaluate yield and similar traits. Thus, it is not surprising that yield is defined based on cumulative effects of different traits.

Using MLM model, the M14/E36-17 and M4/E10-19 markers have demonstrated stable and significant associations with the seed, forage and biological yields under normal and salinity-stress conditions, simultaneously. Most of the functional genes in a genome might have direct or indirect relationships with the yield. Moreover, most of the reported QTLs and identified effective genes attributed to the yield show the pleiotropic effects with more than a single trait (Slafer et al., 2003).

In conclusion according to the association analysis of the phenotypic data, genotypes had significant differences in terms of the investigated traits. Moreover, most of the traits were influenced by the environment and genotypexenvirnment interaction under both normal and salinity-stress conditions. These results indicated the presence of a wide range of diversity in the investigated germplasm of foxtail millet which would facilitate the selection of salinity- resistance genotypes. Therefore, conducting association analysis in different environments would be highly significant. Based on the results obtained via the association analysis of salinity-related traits of foxtail millet, most of the markers affecting the traits under normal and salinity- stress conditions demonstrated acceptable levels of polymorphism and diversity as the population structure and kinship analysis improved. Moreover, the primer combinations used in this investigation had a high polymorphic percentage. Their PIC, MI and the Shannon's indices were also highly reliable. Therefore, the primer combinations employed in this investigation can be considered a powerful tool to distinguish foxtail millet genotypes. The markers used in this investigation had strong effects with highly significant P-value. Therefore, the introduced markers which had significant associations with important agronomic traits could be suitable candidates for further study in order to marker-assisted breeding to improve salinity-resistance genotypes of foxtail millet in arid and semiarid areas. 


\section{Acknowledgement}

The authors would like to appreciate the support provided by the Research and Technology Institute of Plant Production (RTIPP) and Iranian Center of excellence for drought and salinity tolerant crops, ShahidBahonar University of Kerman, Iran and Vice Chancellor for Research and Technology, University of Zabol, Iran.

Funding: Not applicable to the type of our presented research.

Conflicts of interest: The authors declare that they have no conflict of interest in the manuscript.

Ethics approval: Not applicable to the type of our presented research.

Consent to participate: Not applicable to the type of our presented research.

Consent for publication: I am corresponding author, on behalf of all co-authors involved in this paper state that the article entitled can be published in the genetic resources and crop evolution journal. Availability of data and material: My manuscript has data included as electronic supplementary material. Code availability: Not applicable to the type of our presented research.

Authors contributions: The original idea and setting up the current research was implemented by G. Mohammadi-Nejad; the major part of research, field evaluation in the locations and genotyping has been conducted by M.Yazdizadeh; Leila Fahmideh has contributed in manuscript revision and experimental design, Data analysis was done by F. Ebrahimi, The Manuscript was edited by Mahmoud Solouki and Babak Nakhoda as research advisors.

\section{References}

Achleitner A, Tinker NA, Zechner E, Buerstmayr H (2008) Genetic diversity among oat varieties of worldwide origin and associations of AFLP markers with quantitative traits. Theoretical and Applied Genetics 117(7):1041-1053.

Ahmed T, Scholz M, Al-Faraj F, Niaz W (2016) Water-related impacts of climate change on agriculture and subsequently on public health: A review for generalists with particular reference to Pakistan. International journal of environmental research and public health 13(11):1051.

Amadou I, Amza T, Shi Y-H, Le G-W (2011) Chemical analysis and antioxidant properties of foxtail millet bran extracts. Songklanakarin Journal of Science \& Technology 1;33(5).

Ang CY, Liu K, Huang Y-W (1999) Asian foods: Science and technology. CRC Press

Aranzana MJ, Kim S, Zhao K, Bakker E, Horton M, Jakob K, Lister C, Molitor J, Shindo C, Tang C (2005) Genome-wide association mapping in Arabidopsis identifies previously known flowering time and pathogen resistance genes. PLoS genetics1(5). 
Archangi A, Heidari B, Mohammadi-Nejad G (2019) Association between seed yield-related traits and cDNA-AFLP markers in cumin (Cuminum cyminum) under drought and irrigation regimes. Industrial Crops and Products 133(1):276-283.

Arora S, Srivastava S (2002) Suitability of millet-based food products for diabetics. Journal of food science and technology (Mysore) 39(4):423-426.

Austin DF (2006) Fox-tail millets (Setaria: Poaceae)-abandoned food in two hemispheres. Economic Botany 60(2):143-158.

Baker R (2003) Millet production. New Mexico State University Coop. Ext Serv, Las Cruces Guide A414, 18.

Berger GL, Liu S, Hall MD, Brooks WS, Chao S, Muehlbauer GJ, Baik B-K, Steffenson B, Griffey CA (2013) Marker-trait associations in Virginia Tech winter barley identified using genome-wide mapping. Theoretical and Applied Genetics 126(3):693-710.

Collard BC, Jahufer M, Brouwer J, Pang E (2005) An introduction to markers, quantitative trait loci (QTL) mapping and marker-assisted selection for crop improvement: the basic concepts. Euphytica 142(1):169196.

d'Ennequin MLT, Panaud O, Toupance B, Sarr A (2000) Assessment of genetic relationships between Setaria italica and its wild relative S. viridis using AFLP markers. Theoretical and Applied Genetics 100(7):1061-1066.

Dadras AR, Sabouri H, Nejad GM, Sabouri A, Shoai-Deylami M (2014) Association analysis, genetic diversity and structure analysis of tobacco based on AFLP markers. Molecular biology reports 41(5):3317-3329.

Dayakar Rao B, Bhaskarachary K, Arlene Christina G, Sudha Devi G, Vilas AT, Tonapi A (2017) Nutritional and health benefits of millets. ICAR_Indian Institute of Millets Research (IIMR): Hyderabad, Indian 112.

Doust AN, Devos KM, Gadberry MD, Gale MD, Kellogg EA (2004) Genetic control of branching in foxtail millet. Proceedings of the National Academy of Sciences 101(24):9045-9050.

Doyle J (1991) DNA protocols for plants. Molecular techniques in taxonomy. Springer (pp. 283-293).

Dwivedi SL, Upadhyaya HD, Senthilvel S, Hash CT, Fukunaga K, Diao X, Santra D, Baltensperge D, Prasad M (2012) Millets: genetic and genomic resources.

Evanno G, Regnaut S, Goudet J (2005) Detecting the number of clusters of individuals using the software STRUCTURE: a simulation study. Molecular ecology 14(8):2611-2620. 
Gebhardt C, Ballvora A, Walkemeier B, Oberhagemann P, Schüler K (2004) Assessing genetic potential in germplasm collections of crop plants by marker-trait association: a case study for potatoes with quantitative variation of resistance to late blight and maturity type. Molecular Breeding 13(1):93-102.

Gupta S, Kumari K, Das J, Lata C, Puranik S, Prasad M (2011) Development and utilization of novel intron length polymorphic markers in foxtail millet (Setaria italica (L.) P. Beauv.). Genome 54(7):586-602.

Gupta SK, Bansal R, Gopalakrishna T (2014) Development and characterization of genic SSR markers for mungbean (Vigna radiata (L.) Wilczek). Euphytica 195, 245-258.

Jun T-H, Van K, Kim MY, Lee S-H, Walker DR (2008) Association analysis using SSR markers to find QTL for seed protein content in soybean. Euphytica 162(2):179-191.

Kapoor R, Evelin H, Mathur P, Giri B (2013) Arbuscular mycorrhiza: approaches for abiotic stress tolerance in crop plants for sustainable agriculture. Plant acclimation to environmental stress. Springer (pp. 359401).

Kumar S, Ambreen H, Murali T, Bali S, Agarwal M, Kumar A, Goel S, Jagannath A (2015) Assessment of genetic diversity and population structure in a global reference collection of 531 accessions of Carthamus tinctorius L.(Safflower) using AFLP markers. Plant molecular biology reporte 33(5):119-1213.

Kumari K, Muthamilarasan M, Misra G, Gupta S, Subramanian A, Parida SK, Chattopadhyay D, Prasad M (2013) Development of eSSR-markers in Setaria italica and their applicability in studying genetic diversity, cross-transferability and comparative mapping in millet and non-millet species. PloS one 8(6), e67742.

Li Y, Wu S (1996) Traditional maintenance and multiplication of foxtail millet (Setaria italica (L.) P. Beauv.) landraces in China. Euphytica 87(1):33-38.

Liang S, Yang G, Ma Y (2010) Chemical characteristics and fatty acid profile of foxtail millet bran oil. Journal of the american oil chemists' society 87(1):63-67.

Mackay I, Powell W (2007) Methods for linkage disequilibrium mapping in crops. Trends in plant science 12(2):57-63.

Mauro-Herrera M, Wang X, Barbier H, Brutnell TP, Devos KM, Doust AN (2013) Genetic control and comparative genomic analysis of flowering time in Setaria (Poaceae). G3: Genes| Genomes| Genetics 3(2):283-295.

Mehrabi AA, Pour-Aboughadareh A, Mansouri S, Hosseini A (2020) Genome-wide association analysis of root system architecture features and agronomic traits in durum wheat. Molecular Breeding 40:1-7.

Mohammadi-Nejad G, Vaezi H, Majidi-Heravan E, Nakhoda B, Darvish-Kajouri F (2017) Field screening for drought tolerance in Setaria italica and Panicum miliaceum millet germplasm from Iran. Indian Journal 
Mohlke KL, Lange EM, Valle TT, Ghosh S, Magnuson VL, Silander K, Watanabe RM, Chines PS, Bergman RN, Tuomilehto J (2001) Linkage disequilibrium between microsatellite markers extends beyond $1 \mathrm{cM}$ on chromosome 20 in Finns. Genome research 11(7):1221-1226.

Morton MJ, Awlia M, Al-Tamimi N, Saade S, Pailles Y, Negrão S, Tester M (2019) Salt stress under the scalpel-dissecting the genetics of salt tolerance. The Plant Journal 97(1):148-63.

Munns R, James RA, Läuchli A (2006) Approaches to increasing the salt tolerance of wheat and other cereals. Journal of experimental botany 57(5):1025-1043.

Munns R, Tester M (2008) Mechanisms of salinity tolerance. Annu Rev Plant Biol 59(2):651-81.

Mwadzingeni L, Shimelis H, Rees DJG, Tsilo TJ (2017) Genome-wide association analysis of agronomic traits in wheat under drought-stressed and non-stressed conditions. PloS one 12(2):e0171692.

Nyquist WE, Baker R (1991) Estimation of heritability and prediction of selection response in plant populations. Critical reviews in plant sciences 10(3):235-322.

Parisseaux B, Bernardo R (2004) In silico mapping of quantitative trait loci in maize. Theoretical and Applied Genetics 109(3):508-514.

Pritchard JK, Stephens M, Rosenberg NA, Donnelly P (2000) Association mapping in structured populations. The American Journal of Human Genetics 67(1):170-181.

Rao BR, Nagasampige MH, Ravikiran M (2011) Evaluation of nutraceutical properties of selected small millets. Journal of Pharmacy and Bioallied Sciences 3(2):277.

Reddy AR, Chaitanya KV, Vivekanandan M (2004) Drought-induced responses of photosynthesis and antioxidant metabolism in higher plants. Journal of plant physiology 161(11):1189-1202.

Roy SJ, Tucker EJ, Tester M (2011) Genetic analysis of abiotic stress tolerance in crops. Current opinion in plant biology 14(3):232-239.

Saha D, Gowda MC, Arya L, Verma M, Bansal KC (2016) Genetic and genomic resources of small millets. Critical Reviews in Plant Sciences 35(1):56-79.

Sakiroglu M, Sherman-Broyles S, Story A, Moore KJ, Doyle JJ, Brummer EC (2012) Patterns of linkage disequilibrium and association mapping in diploid alfalfa (M. sativa L.). Theoretical and Applied Genetics 125(3):577-590.

SAS Institute (2004) 'SAS/STAT user’s guide.' (SAS Institute: Cary, NC,USA).

Shi J, Li R, Qiu D, Jiang C, Long Y, Morgan C, Bancroft I, Zhao J, Meng J (2009) Unraveling the complex trait of crop yield with quantitative trait loci mapping in Brassica napus. Genetics 182(3):851-861. 
Singh RK, Jaishankar J, Muthamilarasan M, Shweta S, Dangi A, Prasad M (2016) Genome-wide analysis of heat shock proteins in $\mathrm{C} 4$ model, foxtail millet identifies potential candidates for crop improvement under abiotic stress. Scientific reports 6(1):1-14.

Slafer G (2003) Genetic basis of yield as viewed from a crop physiologist's perspective. Annals of Applied Biology 142(2):117-128.

Vos P, Hogers R, Bleeker M, Reijans M, Lee Tvd, Hornes M, Friters A, Pot J, Paleman J, Kuiper M (1995) AFLP: a new technique for DNA fingerprinting. Nucleic acids research (21):4407-4414.

Yazdizadeh M, Fahmideh L, Mohammadi-Nejad G, Solouki M, Nakhoda B (2020) Association analysis between agronomic traits and AFLP markers in a wide germplasm of proso millet (Panicum miliaceum L.) under normal and salinity stress conditions. BMC plant biology 20(1):1-8.

Yu J, Pressoir G, Briggs WH, Bi IV, Yamasaki M, Doebley JF, McMullen MD, Gaut BS, Nielsen DM, Holland JB (2006) A unified mixed-model method for association mapping that accounts for multiple levels of relatedness. Nature genetics 38(2):203-208.

Zhang C, Zhang H, Li J (2007) Advances of millet research on nutrition and application. Journal of the Chinese Cereals and Oils Association 22(1):51-55.

Zhang J-P, Zhang J-P, Liu T-S, Zhang J-P, Liu T-S, Zheng J, Zhang J-P, Liu T-S, Zheng J, Jin Z (2007) Cloning and characterization of a putative 12-oxophytodienoic acid reductase cdna induced by osmotic stress in roots of foxtail Millet: Full length research paper. DNA Sequence 18(2):138-144.

Zhang, C., Zhang, H., \& Li, J. X. (2007). Advances of millet research on nutrition and application. J Chinese Cereals Oils Assoc, 22, 51-55.

Zhu C, Gore M, Buckler ES, Yu J (2008) Status and prospects of association mapping in plants. The plant genome 1(1):5-20.

\section{Figures}



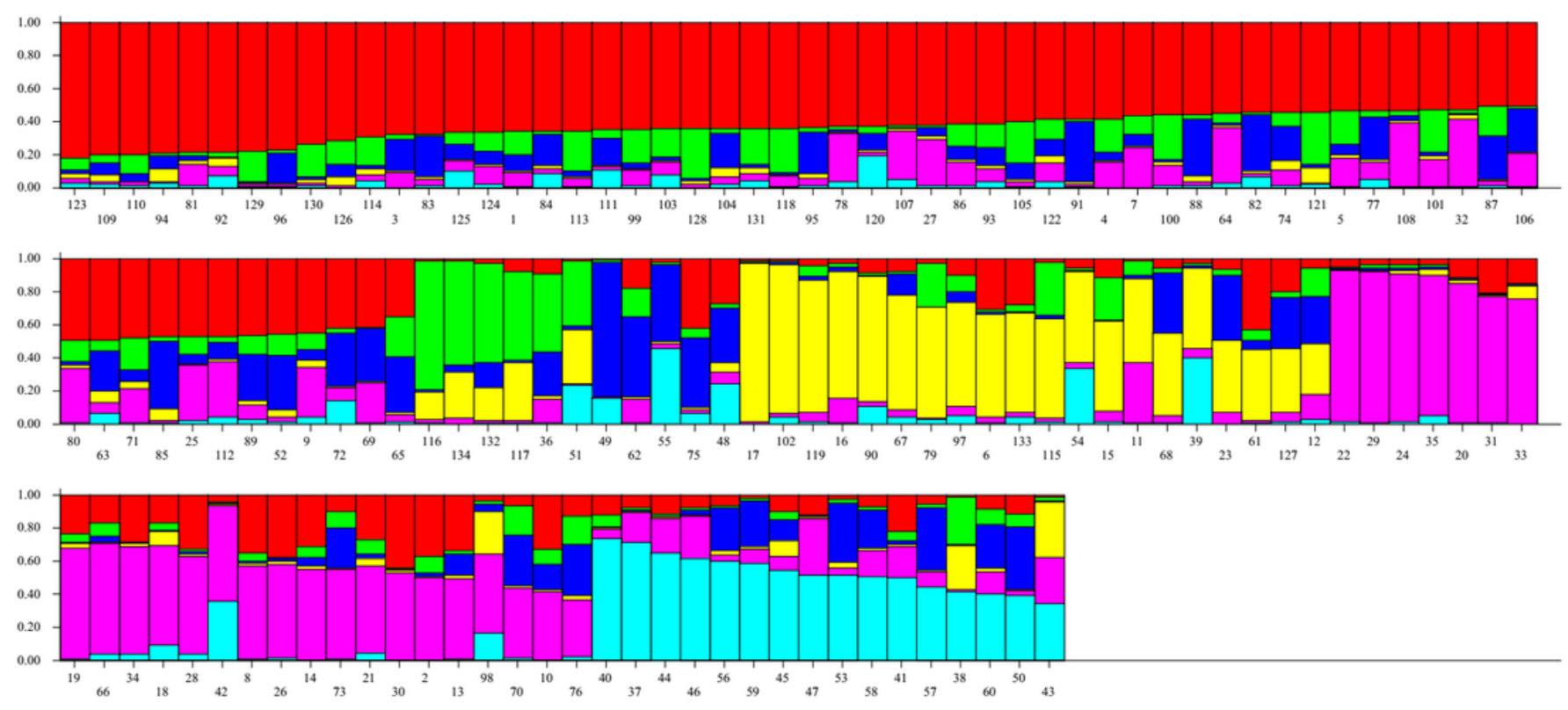

\section{Figure 1}

Biplot based on data obtained from 12 AFLP primer combinations using the structure software, red, green, yellow, dark blue, light blue and violet colors indicate foxtail genotypes divided into six subpopulations.

\begin{tabular}{rcccccc}
\hline K & Reps & Mean LnP(K) & Stdev LnP(K) & $\operatorname{Ln}^{\prime}(\mathbf{K})$ & $\left|\operatorname{Ln}{ }^{\prime \prime}(K)\right|$ & Delta K \\
2 & 5 & -22195.160000 & 47.545957 & - & - & - \\
3 & 5 & -21774.880000 & 53.116542 & 420.280000 & 361.920000 & 6.813697 \\
4 & 5 & -20992.680000 & 17.268237 & 782.200000 & 678.120000 & 39.269788 \\
5 & 5 & -20888.600000 & 222.248453 & 104.080000 & 198.340000 & 0.892425 \\
6 & 5 & -20586.180000 & 20.780568 & 302.420000 & 842.920000 & 40.562895 \\
7 & 5 & -21126.680000 & 1549.598692 & -540.500000 & 538.260000 & 0.347354 \\
8 & 5 & -22205.440000 & 3148.359671 & -1078.760000 & 1397.800000 & 0.443977 \\
9 & 5 & -24682.000000 & 8590.881382 & -2476.560000 & 6571.700000 & 0.764962 \\
10 & 5 & -33730.260000 & 12960.851430 & -9048.260000 & - & -
\end{tabular}

Figure 2

The results of Evanno's method to determine the $\mathrm{K}$ values, The yellow colored row represents $\mathrm{K}$ at maximum values of $\Delta \mathrm{K}$. 
$L(K)$



Figure 3

The graph $(L(K), K)$ of Evanno's method to determine the $K$ values. 


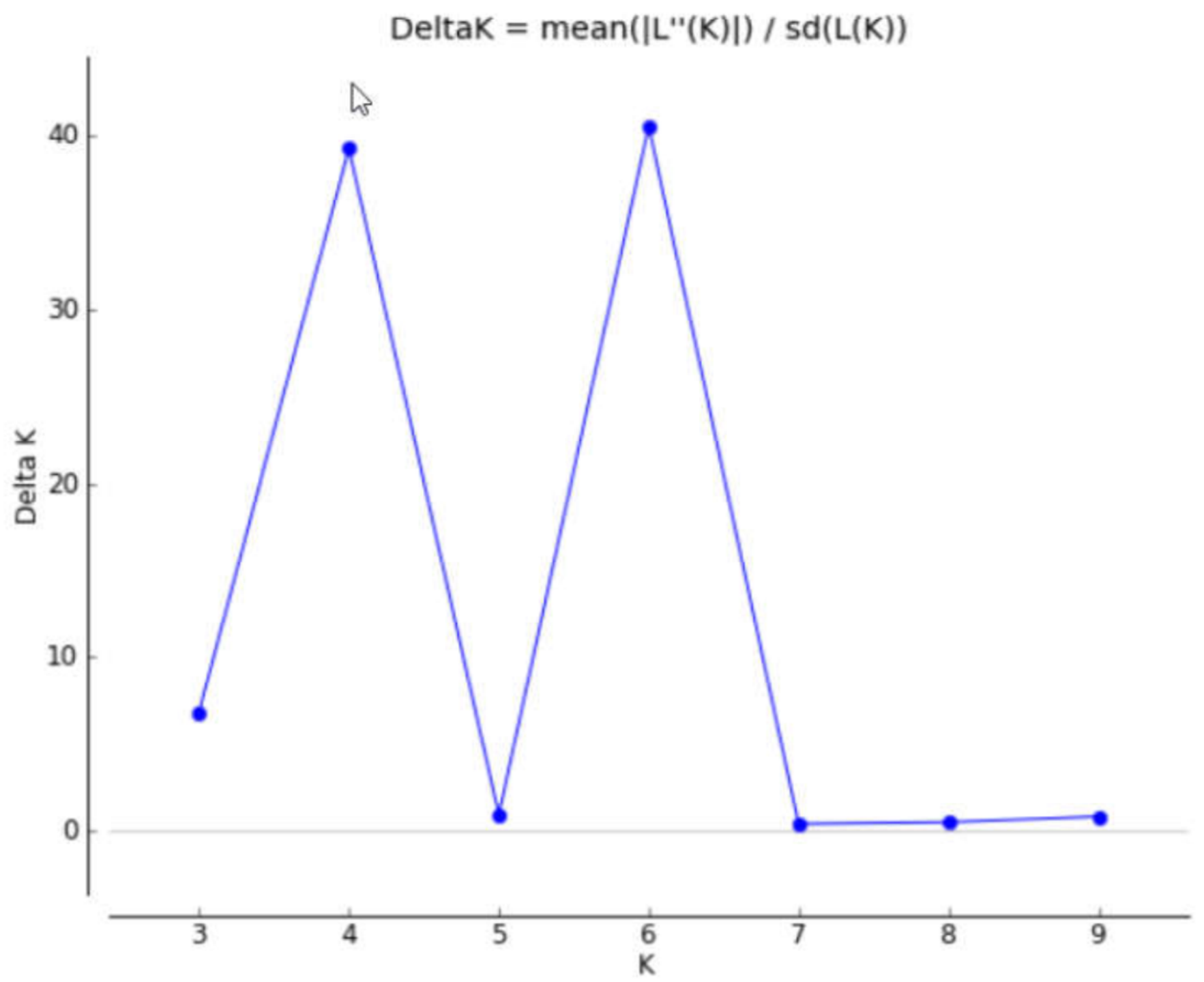

Figure 4

The graph (Delta K, K) of Evanno's method to determine the K values.

\section{Supplementary Files}

This is a list of supplementary files associated with this preprint. Click to download.

- SupplementalTable1.docx

- SupplementalTable2.docx

- SupplementalTable3.docx 